MILITARY TECHNICAL COLLEGE CAIRC - EGYPT

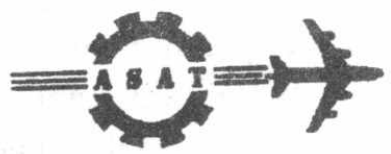

$7^{\text {th }}$ INTERNATIONAL CONF. ON AEROSPACE SCIENCES \& AVIATION TECHNOLOGY

\title{
A CONCEPTUAL GAMMA SHIELD DESIGN USING THE DRP MODEL COMPUTATION
}

\author{
ENSHERAH E. AHMED*, F. A. RAHMAN**
}

\begin{abstract}
The purpose of this investigation is to assess the basic areas of concern in the development of reactor shielding conceptual design calculations. A spherical shield model composed of two concentric spherical zones of low carbon steel and lead have been constructed to surround a Co-60 gamma point source. Two alternative configurations have been considered in the model computations. The numerical calculations have been performed using both the ANISN code and the DRP model computations together with the DLC 75-Bugle 80 data library. A resume of results for deep penetration in different shield materials with different packing densities is presented and analyzed. The results showed that the gamma fluxes attenuation is increased with increasing the packing density of the shield material which reflects its importance of considering it as a safety parameter in shielding design.
\end{abstract}

\section{KEY WORDS}

Safety limits , conceptual design , $\gamma$-shielding , ANISN- Code , DRP-Code.

\section{INTRODUCTION}

One of the most difficult problems in shielding design analysis for the effective reactor shielding is that of considering the transmission of shield materials containing voids or relatively poor attenuation characteristics. Such irregularities could be gaps, ducts, inhomogeneous materials,... etc. Exactly, as voids increase the effective relaxation length for hard radiation, they also increase the diffusion length for low radiation energy. In shielding design, the inhomogenity could be resulted from preparation, moulding, casting, thermal treatment,......etc. during the fabrication of shielding materials or sometimes the shield

* Reactors Dept., Nuclear Research Center (NRC), Atomic Energy Authority(AEA), Cairo , Egypt. ** Operational Safety Dept., National Center of Nuclear Safety and Radiation Control (NCNSRC), AEA , Cairo, Egypt. 
material could be utilized in torm of pebbles. Thus in shielding design analysis, the inhomogeneous materials - as for example a pebble shield - are considered to consist of randomly packed collection of pieces of shielding materials. So, it is required to consider the transmission of a shield to contain many closely spaced voids. In the limit, in which the average distance between voids vanishes relative to the relaxation length, the increase in transmission is given simply by the reduced density effect, according to a certain factor. When the pebbles are not negligibly small, there will be significant statistical variations in the distance through the solid shield material traversed by the gamma-ray through the shield. The effect of such these poor attenuation regions in solid shield materials can usually be calculated by one of several approximate methods. One of the most useful methods for calculating the gamma-ray fluxes attenuation through the distributed voids in shields is that one for treating voids as perturbations via the one velocity transport equation [1].

\section{GEOMETRICAL AND PHYSICAL MODELS}

The geometrical model consists of two concentric spheres with a fixed Co- 60 point $\gamma$-ay source located at its center. The inner sphere is $20 \mathrm{~cm}$. outer radius of low carbon steel (contains small amount of impurities which do not affect the $\gamma$-ray attenuation). The outer spherical shell is $20 \mathrm{~cm}$. thickness of lead (Pb). Thus, this configuration has two zones. The model also comprises two alternative configurations; configuration I (with Fe has different values of packing densities while $\mathrm{Pb}$ is void free) and configuration II (with $\mathrm{Fe}$ is void free while $\mathrm{Pb}$ has different values of packing densities). The $\gamma$-ray source energy spectra is divided into 21 energy group structure [2,3]. The dense random packing DRP of hard spheres model, which is a good model to describe the structure of metals, was used by distributing atoms in a randomly irregular tetrahedron form [4]

\section{METHOD OF ANALYSIS}

The energy dependence Boltzmann transport equation [5] is solved numerically. The energy dependence is discretized in the usual multigroup approximation. The angular variable and the angular dependence are discretized in the discrete ordinates approximation (S6 ). However, the angular dependence of the scattering cross -section is represented by the standard orthogonal Legendre polynomial approximation (P3). The spatial operator and the spatial dependence are discretized in the finite difference approximation. The multigroup finite difference discrete equations are then solved iteratively $[6,7]$. In the DRP model, the depicting atoms are packed together in a cluster which is dense because it contains no vacancies large enough to accommodate : another atom and is random because it lacks long-range order [8]. The detailed structure of random packing depends on many factors of modeling; softness and nature of the interatomic potential, nature of the boundary and the preparation conditions or the packing algorithms. The packing destiny $\eta$ is given by [4]:

$$
\eta=\zeta^{3} \pi H / 6 L^{3}
$$

with $\zeta$ can be calculated from: 


$$
H(R)=(1 / H c) \quad \sum_{i=1}^{H c} \sum_{j=1}^{H} \xi\left(R-\left|R_{i}-R_{j}\right|\right) \quad \ldots \ldots \ldots . . . . i \# j
$$

where $\zeta$ is the atomic diameter, $L$ is the side of the lattice cube, $R$ is the maximum radius of the cluster, $R i$ and $R j$ are the maximum distance between the center of the globe and the center of atoms $i$ and $j, H$ is the total no. of distributing atoms in the cluster, $H(R)$ is its statistical variation within the cluster and $H c$ is the no. of the central atoms [4].

The resulting $\eta$ is used to calculate the actual density of shielding materials by multiplying it by the corresponding theoretical density which in turns used to initiate a data library for the 21 groups of Po to P3 macroscopic cross sections for the ANISN code. This was done for all investigated values of $\eta(0.35$ to 1.0$)$.

\section{RESULTS AND DISCUSSIONS}

The 21 energy groups $\gamma$-radial fluxes distributions through the spherical shield configurations were calculated ( for P3 approximation and S6 angular quadrature). The active $\gamma$-energy range up to $14 \mathrm{Mev}$ was divided into 21 groups [3]. The set of the physical constants were evaluated using both DLC-75, Bugle-80 cross sectionis data library [2] and the DRP code [4].

The calculated $\gamma$-rays radial fluxes attenuations for energy groups number 11 to 21 ( 1.0 to $0.01 \mathrm{Mev}$ ) through both configurations I and II for different packing densities are shown in Fig.1. Figure 1(a) presents such attenuation gradients when both $\mathrm{Fe}$ and $\mathrm{Pb}$ spherical shield shells are totally void free (i.e. $\eta=1.0$ for each), while Fig.1 (b,c) illustrate the attenuation behaviour in both configurations I \& II when $\eta$ is reduced to 0.35 . It is clearly observed that the attenuation intensities increase with increasing the shield radius. Also, a rapid attenuation behaviour for $\gamma$-rays upon entering the shield (shallow penetration) is clearly noticed from the figures. This may be attributed to the geometrical spreading phenomena. As also clearly seen, all values of $\gamma$-flux intensities in Fig.1 (a) are less than the corresponding values of Fig.1 (b,c). This means more attenuation characteristics is attained by increasing $\eta$ to 1.0 . Besides, as seen from the figures, the attenuation behaviour through configuration II is sharper than that through configuration I since all energy groups are totally attenuated at shield radius $<30 \mathrm{~cm}$. in configuration II whereas they exceed $40 \mathrm{~cm}$. shield radius in configuration I.

The effect of the variation of the packing density of Fe on the calculated $\gamma$-fluxes of energy groups no. 11 ( $1.0-0.8 \mathrm{Mev})$, no. $16(0.2-0.1 \mathrm{Mev})$ and no. $20(0.02-0.01 \mathrm{Mev})$ for configuration I are shown in Fig.2 ( $\mathrm{a}, \mathrm{b}, \mathrm{c})$. It is clearly seen from the figures that the fluxes distributions showed a similar attenuation behaviour for all values of packing densities. The figure also shows that attenuation intensities within both shield layers are increased with increasing the packing density for both middle and deep penetrations for the three energy groups ( no. 11,16 and 20). This may be attributed to the effect of both mean free path and diffusion length.

The effect of $\mathrm{Fe}$ packing density variation ( $\eta_{\mathrm{Fe}}$ ) on the calculated $\gamma$ - fluxes of energy groups no.11,16 and 20 respectively at different penetrating redii are shown in Fig. 3 (a,b,c). As seen 


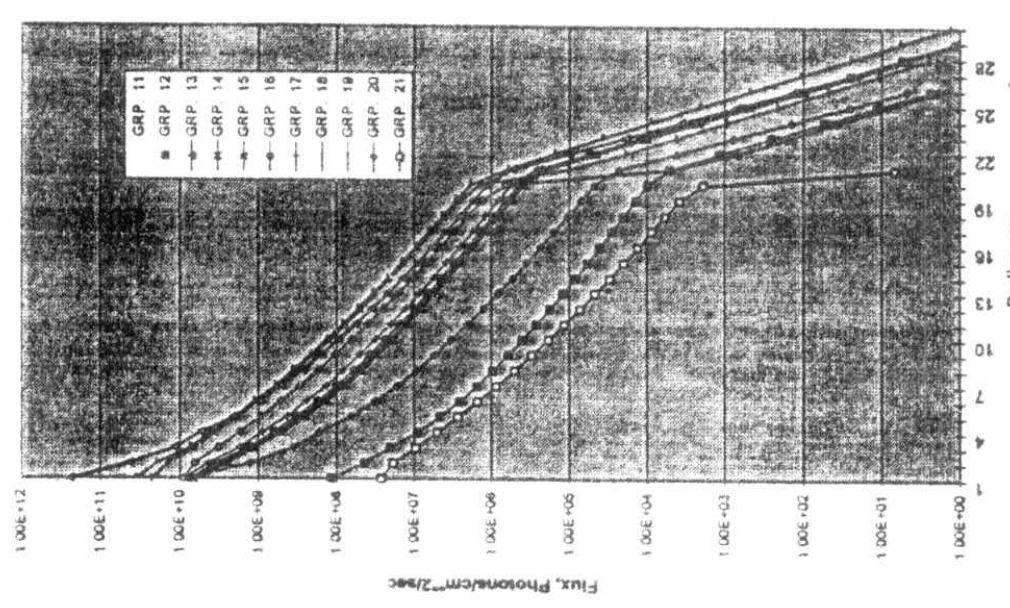

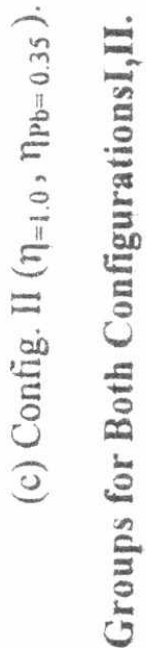
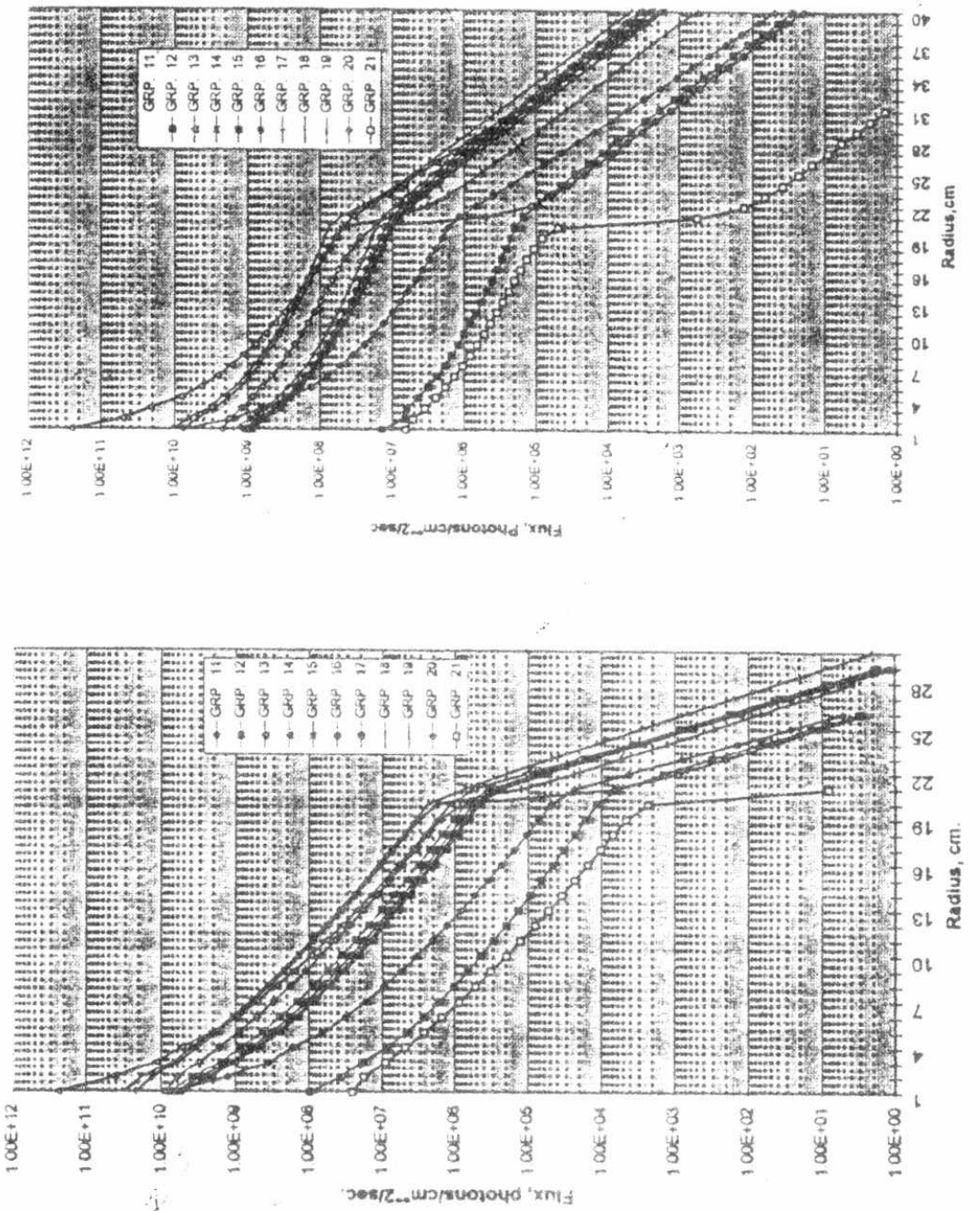

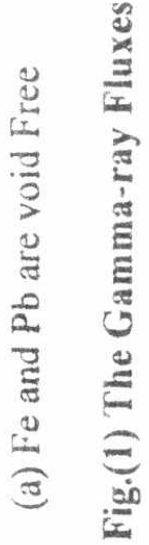



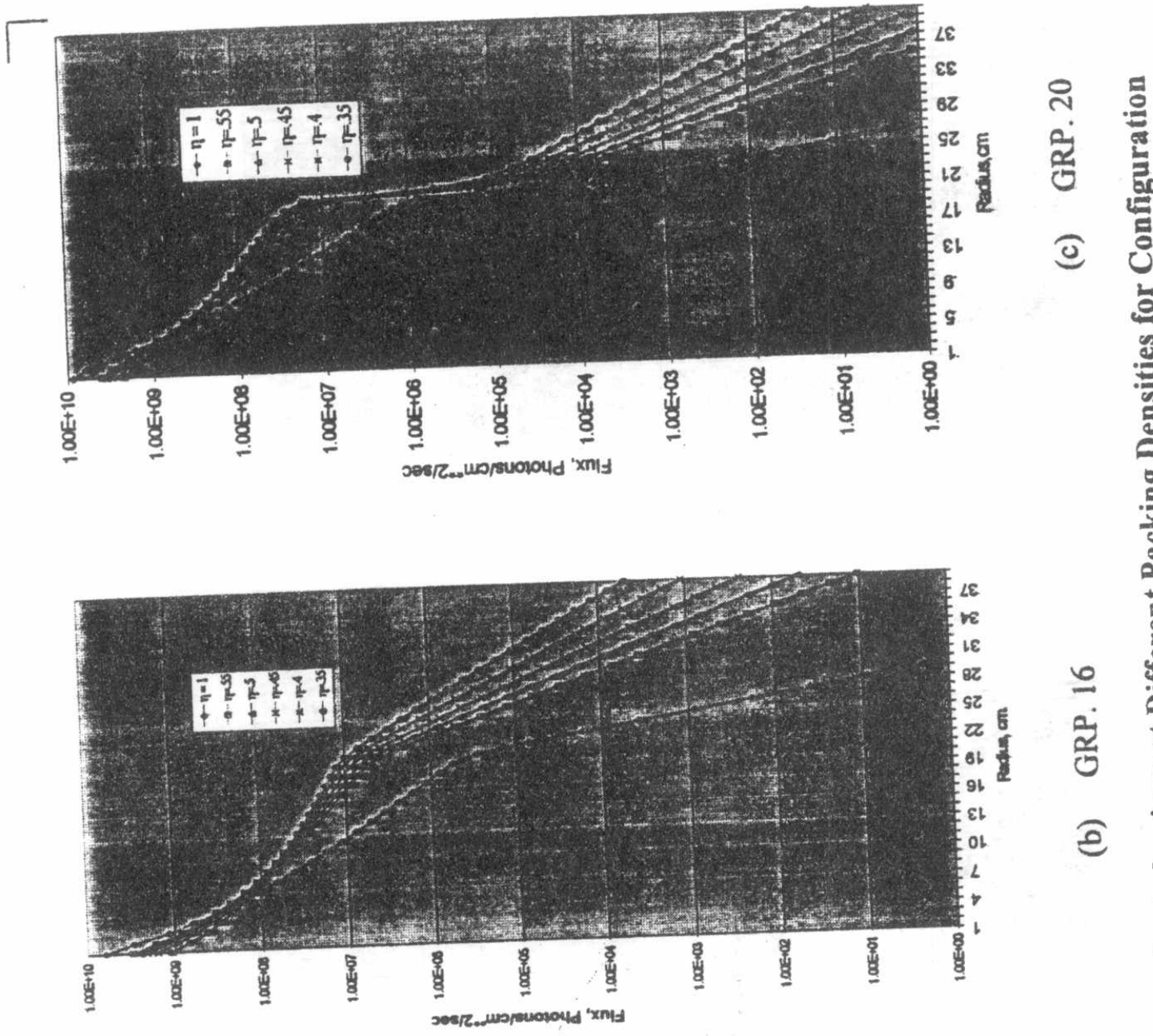

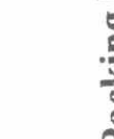

:

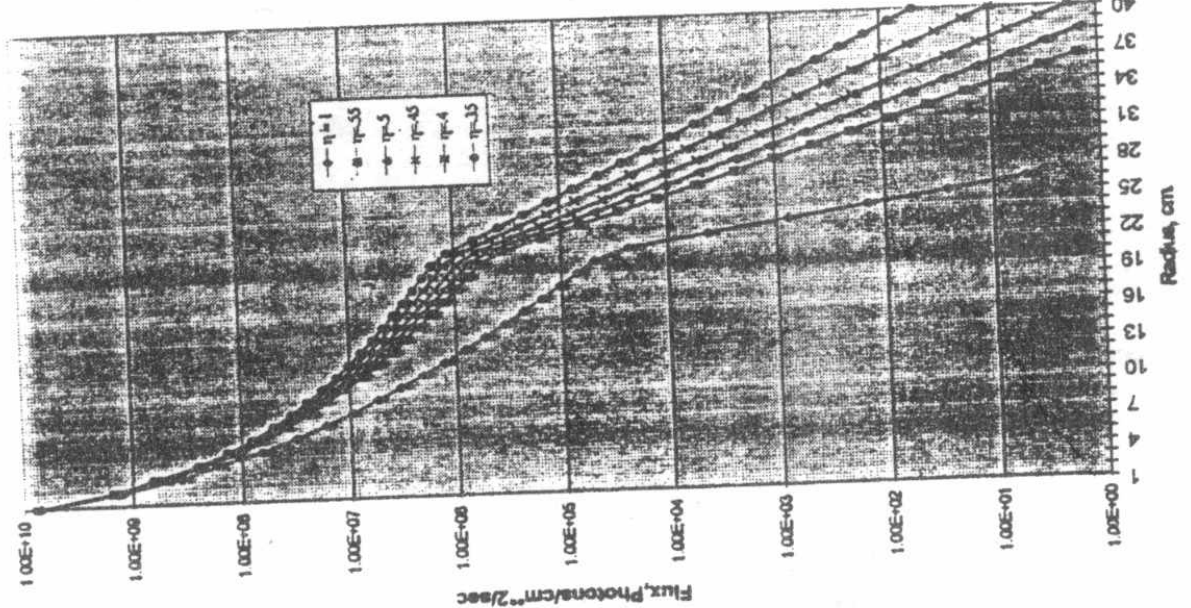

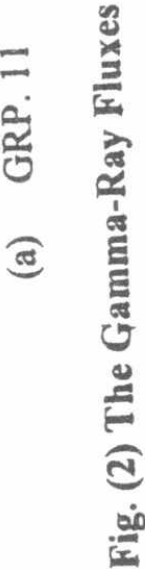




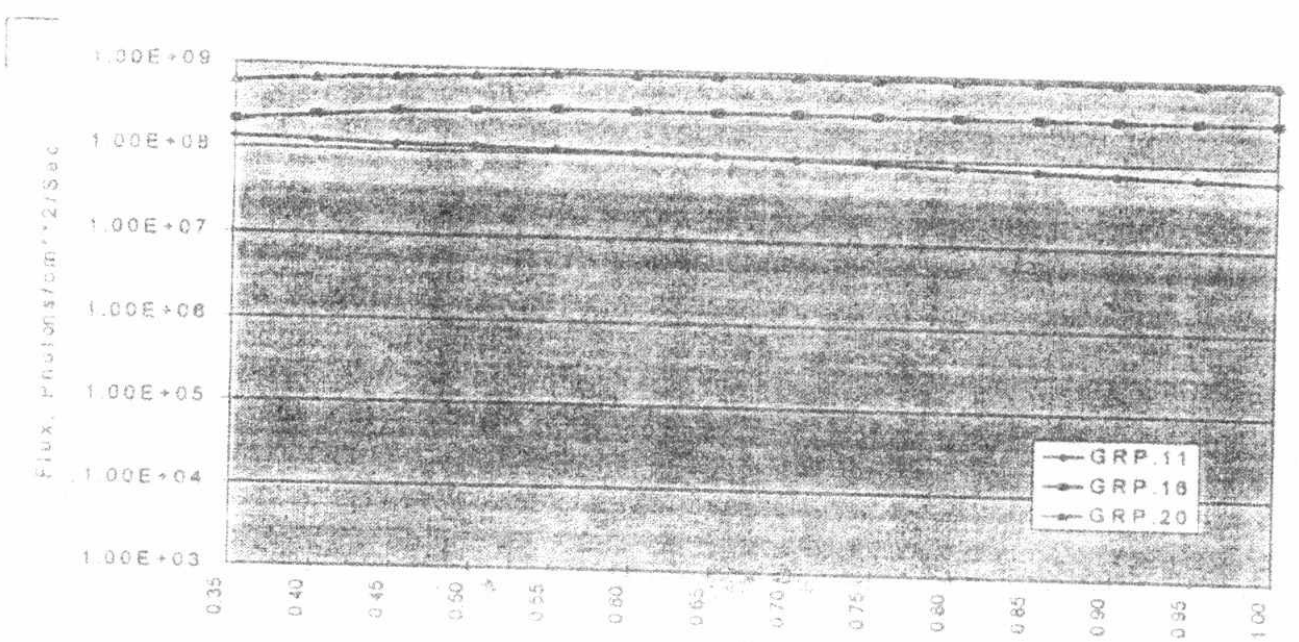

(a) Shallow Penetration $(r=5 \mathrm{~cm})$

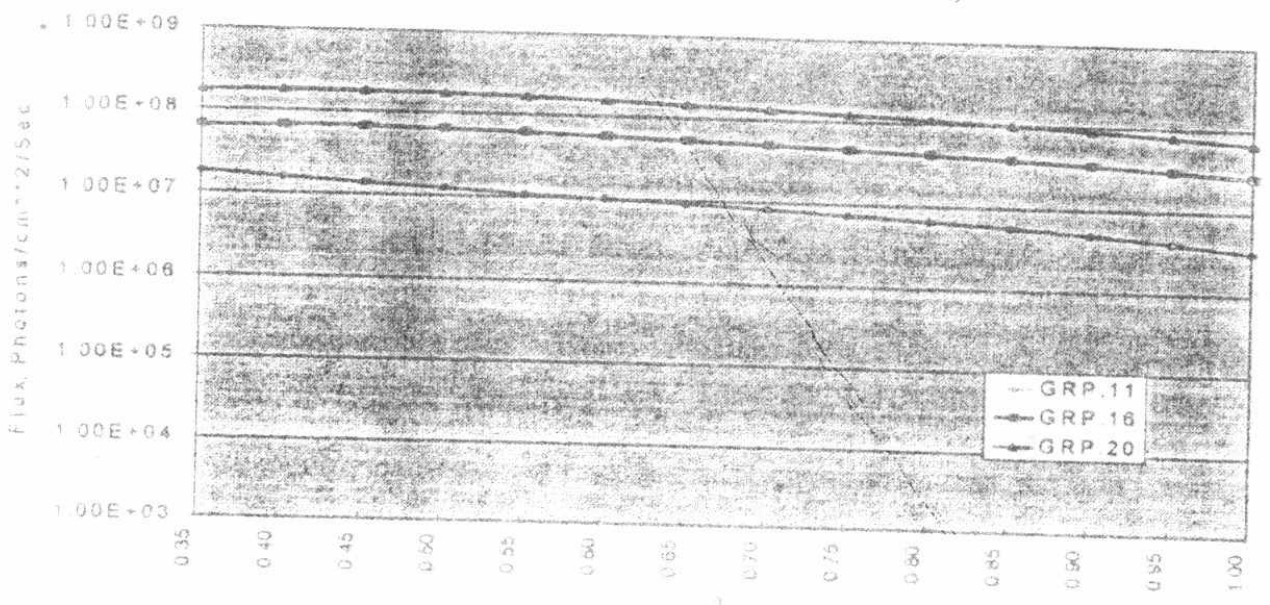

(b) Middle Penetration $(x=10 \mathrm{~cm})$

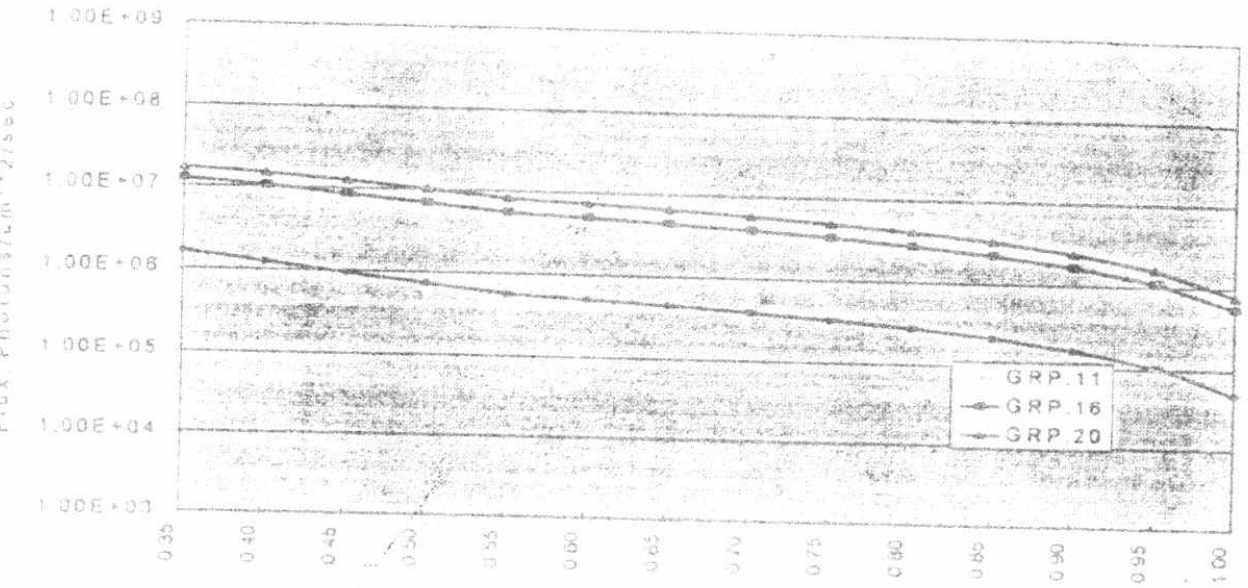

(c) Deep Penetration $(r=20 \mathrm{~cm}$ )

Fig (3) The Effect of $\eta_{\mathrm{Fe}}$ on $\phi$, Penetrarion at different radii for energy groups no. 11,16 and 20 . 
from the Figs. 2 and 3 ; at shallow penetration $(r=5 \mathrm{~cm}$. ) the fluxes distributions increase as $\eta_{\mathrm{Fe}}$ increase up to 1.0 for the low energy groups (no. 20,16 ) whereas it decreases for the high energy group (no.11). This behavioural change may be attributed to the mode of $\gamma$-rays interaction with matter during its penetration within the shield since they interact through separate processes. However, the effectiveness of shield materials in attenuating $\gamma$-rays is determined by the combined effect of a succession of processes upon the over-all propagation of $\gamma$-ray of those processes. For the proposed shield materials and for low energy $\gamma$-rays, the photoelectric effect is predominant. Also, the probability of rayleight scattering is enhanced and prevails greatly. For high photon energies, the effect of compton scattering and pair production is the predominant. The figures also showed that at middle $(\mathrm{r}=10 \mathrm{~cm}$. $)$ and deep penetrations $(r=20 \mathrm{~cm}$. ), the gamma flux distribution decrease as going through the shield shells with increasing $\eta_{\mathrm{Fe}}$ for the three energy groups. This confirms the importance of producing high quality grade iron as a shielding material since it greatly affects its packing density and implies the imortance of considering $\eta$ as a controlling factor for gamma attenuation. In other words, this investigation recommends to introduce a void percentage stamp for shielding materials for the sake of precise prediction of the dimensional configuration needed for the requirements of safety limits calculations.

\section{CONCLUSIONS}

1- The calculated gamma fluxes distributions and its energy spectra for all attenuations through both iron and lead spherical shield layers have revealed that the use of multigroup cross-section data (DLC-75, Bugl-80)with the angular scattering represented by $\left(\mathrm{P}_{3}\right)$ Legendre expansion and the DRP model are adequate for predicting the variation in these distributions induced due to variation of packing density.

2- The variation in the gamma fluxes attenuation distributions induced by the variation of packing density in lead is less than that in iron.

3- The gamma fluxes attenuation is increased with increasing the shield material packing density which reflects its importance in considering it as an important safety parameter in shielding design.

4- For the geometrical model considered in this investigation, the effect of packing density variation in controlling the gamma rays attenuations is confirmed in both configurations for middle and deep penetrations.

5- The investigation recommends to introduce a void percentage stamp for shielding materials for the sake of precise prediction of the dimensional configuration needed for the requirements of the safety limits calculations.

\section{REFERENCES}

[1] Bell, G.I. and Glasstone, S.; Nuclear Reactor Theory, Van Nostrand Co., N.Y. (1990).

[2] ORNL; "RISC Data Library Collection DLC-75/ Bugle 80", ORNL and ANS- 6.1.2., USA (1985).

[3] Rahman, F. A.,et al; “ATheoretical Model for Gamma Ray Attenuation and Distributionin a Laminated Shield”, Int. J. Rad. Appl. Instrum., Part A, England (1989).

[4] El - Desouki , S.F.;" Study of Some Physical Properties of Some Non- crystalline

Compounds", Ph. D. Thesis, Fac. of Sc. , Al-Azhar Univ. ,Cairo (1988).

[5] Bartine , D.E.; " Proc. of 6 th. Int. Conf. on Radiation Shielding ", Tokyo (1983). 
[6] Larsen ,E.W.;"Diffusion -Synthetic Acceleration Method for Discrete - Ordinate Problems", Transport Theory and Statistical Physics, 13 ,pp 107-126 (1984).

[7] Englew,w. and Mynatt, F.R. ;" A Comparison of Two Methods of Iteration Convergence Acceleration in Discrete Ordinates Codes", Trans. ANS ,11 ,pp 193-194,(1969).

[8] Finney, J.L and Luborsky, F.E.(Ed.); Amorphous Metallic Alloys, Butterworth Co. Pub. Ltd., p. 42 (1983). 